\title{
Fast and accurate computation of the Weber parabolic cylinder function $W(a, x)$
}

\author{
Amparo Gil \\ Departamento de Matemática Aplicada y CC. de la Computación. \\ ETSI Caminos. Universidad de Cantabria. 39005-Santander, Spain. \\ Javier Segura \\ Departamento de Matemáticas, Estadística y Computación, \\ Univ. de Cantabria, 39005 Santander, Spain. \\ Nico M. Temme \\ CWI, Science Park 123, 1098 XG Amsterdam, The Netherlands. \\ e-mail: amparo.gil@unican.es, javier.segura@unican.es, nicot@cwi.nl
}

March 27, 2010

\begin{abstract}
Methods for the numerical evaluation of the Weber parabolic cylinder functions $W(a, \pm x)$, which are independent solutions of the inverted harmonic oscillator $y^{\prime \prime}+\left(x^{2} / 4-a\right) y=0$, are described. The functions appear in the solution of many physical problems, and notably in quantum mechanics. It is shown that the combined use of Maclaurin series, Chebyshev series, uniform asymptotic expansions for large $a$ and/or $x$ and the integration of the differential equation by local Taylor series are enough for computing accurately the functions in a wide rage of parameters. Differently from previous methods, the computational scheme is stable in the sense that high accuracy is retained: only 2 or 3 digits may be lost in double precision computations.
\end{abstract}

Keywords \& Phrases: Weber parabolic cylinder functions; inverted harmonic oscillator; evaluation of special functions; Chebyshev expansions; Taylor methods for differential equations; asymptotic analysis. 


\section{Introduction}

Parabolic cylinder functions (PCFs) $U(a, x), V(a, x)$ and $W(a, x)$ are useful functions appearing in many scientific problems, and in particular in many physical applications. As an indication of the relevance of this set of functions, the search "parabolic cylinder function" gives more that 2,000 hits in Google Scholar. In particular, the parabolic cylinder function $W(a, x)$ appears, for example, in the analysis of the wave scattering by a parabolic cylinder [?], in astrophysics [?, ?], in the study of quantum mechanical problems such as quantum tunnelling, particle production in electric and magnetic [?, ?, ?] and gravitational fields [?], atomic physics [?, ?], semiconductor physics [?] and many more.

As a relatively simple example problem involving $W(a, x)$, consider solving the Schrödinger equation

$$
-\frac{\hbar^{2}}{2 m} \psi_{x x}+V(x) \psi(x)=i \hbar \psi_{t}
$$

with $V(x)$ a parabolic potential $V(x)=\alpha-x^{2} / 4, \alpha>0$, also known as inverted harmonic oscillator. We consider an energy eigenstate $\psi(x, t)=$ $N u(x) e^{-i E t / \hbar}$ and arrive to the time independent Schrödinger equation (setting $\hbar^{2} / 2 m=1$ for simplicity)

$$
u_{x x}+\left(x^{2} / 4-a\right) u=0, a=\alpha-E
$$

The solutions of this equation can be written in terms of the parabolic cylinder functions $W(a, \pm x)$. In particular, the functions

$$
\Phi(a, x)=N\left[k(a)^{-1 / 2} W(a, x)+i k(a)^{1 / 2} W(a,-x)\right],
$$

where

$$
k(a)=\left(\sqrt{1+e^{2 \pi a}}+e^{\pi a}\right)^{-1}, N=\left(4 \pi\left(1+e^{2 \pi a}\right)\right)^{-1 / 2},
$$

represent normalized energy eigenstate coming from the left (see Figure 1, right) and transmitting to the right (tunnelling the potential barrier in a classically forbidden regime when $a>0$ ). Reference [?] describes in detail the quantum mechanics of the inverted harmonic oscillator.

The functions $W(a, x)$ are part of a wider set of functions known as Parabolic Cylinder functions. The standard differential equations having PCFs as solutions have the following two forms:

$$
y^{\prime \prime}-\left(\frac{1}{4} x^{2}+a\right) y=0 \quad x \geq 0,
$$


which has $\{U(a, x), V(a, x)\}$ as a numerically satisfactory pair of solutions ${ }^{1}$ and

$$
y^{\prime \prime}+\left(\frac{1}{4} x^{2}-a\right) y=0, \quad x \geq 0,
$$

which has $\{W(a, x), W(a,-x)\}$ as a satisfactory pair.

For the first case, double precision algorithms are known, starting with the method based on asymptotics and recursions of [?]. More recently, algorithms using asymptotics and numerical quadrature and, to a lesser extent, recurrence relations, were developed for computing $U(a, x), V(a, x)$ and scaled $U$ and $V$ functions [?, ?] (computable for unrestricted values of the variables).

The situation for the functions $W(a, \pm x)$ is, up to the date, much less satisfactory. In [?], complex recurrence relations and the integration of auxiliary functions by means of Runge-Kutta methods for intermediate values of the variables produced simple precision accuracy when doing the computations in double precision. The situation was not improved in [?], where the typical accuracy is 6 digits when computing with 14D. More recently, integral representations were derived in [?], but the application is more difficult than in the case of $U(a, x)$ and $V(a, x)$, because some numerical instabilities arise which need special treatment (see [?, §4.3.3]).

No available algorithms exist for the accurate computation of the $W(a, x)$ for real $a$ and $x$, although packages such as Maple and Mathematica can compute such functions through their relation with the complex parabolic cylinder function $U\left(i a, x e^{-i \pi / 4}\right)$ (see Eq. (2.5)) or, equivalently, through their relation with confluent hypergeometric functions (see [?], equations 19.7.1 and 19.25.1 ${ }^{2}$ ). However, this is an unsatisfactory method of computation, first because a real function is computed using complex arithmetic and second because strong cancellations take place in the computation of $W(a, x), x>0$.

In this paper we compute the parabolic cylinder function $W(a, x)$ by Maclaurin and Chebyshev series for small values of $|a|$ and $|x|$, asymptotic expansions for large $|a|$ and/or $|x|$ and integrating the differential with using local Taylor series for moderate values of $|a|$ and $|x|$. As we will see, these ingredients suffice for computing the parabolic cylinder functions $W(a, x)$ for real $a$ and $x$ with a relative accuracy better than $510^{-13}$.

\footnotetext{
${ }^{1}$ Numerically satisfactory in the sense of Miller [?, ?]

${ }^{2}$ We notice that the \pm sign in this formula should be $\mp$ instead
} 
Figure 1: Left: The function $W(a, x)$ for $a=0.5$ and $x \in[-20,20]$ is plotted. The parabola $y=a-x^{2} / 4$ is also shown. Right: The amplitude $|\Phi(a, x)|$ of the left-incoming waves (Eq. (1.3)) as a function of $x$ and for three values of $a: a=-0.5$ (solid line), $a=0$ (dashed line) and $a=0.5$ (dotted line). The larger $a$ is, the smaller the amplitude transmitted to the right of the barrier.

\section{Some properties of the $W$-function.}

The functions $W(a, x)$ and $W(a,-x)$ are two linearly independent solutions of the differential equation (1.6). For $a<0$ the solutions oscillate on the real $x$-axis; for $a>0$ there are turning points at $\pm 2 \sqrt{a}$, and the oscillations occur outside the interval $[-2 \sqrt{a}, 2 \sqrt{a}]$ (see Figure 1, left).

The solutions $W(a, x)$ and $W(a,-x)$ constitute a numerically satisfactory pair for $-\infty<x<\infty$; see [?, ?]. Initial values are (for these and further details, see [?, pp. 692-697])

$$
W(a, 0)=2^{-\frac{3}{4}}\left|\frac{\Gamma\left(\frac{1}{4}+\frac{1}{2} i a\right)}{\Gamma\left(\frac{3}{4}+\frac{1}{2} i a\right)}\right|^{\frac{1}{2}}, \quad W^{\prime}(a, 0)=-2^{-\frac{1}{4}}\left|\frac{\Gamma\left(\frac{3}{4}+\frac{1}{2} i a\right)}{\Gamma\left(\frac{1}{4}+\frac{1}{2} i a\right)}\right|^{\frac{1}{2}} .
$$

The Wronskian of $W(a, x)$ and $W(a,-x)$ is

$$
\mathcal{W}[W(a, x), W(a,-x)]=1 .
$$

Observe that the Wronskian relation means

$$
W(a, x) \frac{d}{d x} W(a,-x)-\frac{d}{d x} W(a, x) W(a,-x)=1,
$$


that is

$$
W(a, x) W^{\prime}(a,-x)+W^{\prime}(a, x) W(a,-x)=-1 .
$$

The relation with the function $U(a, x)$ reads

$$
\frac{1}{\sqrt{k(a)}} W(a, x)+i \sqrt{k(a)} W(a,-x)=\sqrt{2} e^{\frac{1}{4} \pi a+i \rho(a)} U\left(i a, x e^{-\pi i / 4}\right),
$$

which follows from using the initial values of the functions, but also from [?, 19.17.6 and 19.17.9]. The quantities $k(a)$ and $\rho(a)$ are given by

$$
k(a)=\sqrt{1+e^{2 \pi a}}-e^{\pi a}=\frac{1}{\sqrt{1+e^{2 \pi a}}+e^{\pi a}},
$$

and

$$
\rho(a)=\frac{1}{8} \pi+\frac{1}{2} \phi_{2}(a), \quad \phi_{2}(a)=\operatorname{ph} \Gamma\left(\frac{1}{2}+i a\right),
$$

the branch being defined by $\phi_{2}(0)=0$ and by continuity elsewhere. In $\S 4$ we will use the auxiliary function $\rho^{*}(a)$ for large $|a|$ :

$$
\rho(a)=\frac{1}{8} \pi-\frac{1}{2} a+\frac{1}{4} a \ln a^{2}+\rho^{*}(a) .
$$

We have $\rho^{*}(a)=\mathcal{O}(1 / a)$ as $a \rightarrow \infty$.

Because we assume that $a$ and $x$, and hence $W(a, \pm x)$, are real, then, using (2.5),

$$
\begin{aligned}
& W(a, x)=\sqrt{2 k(a)} e^{\frac{1}{4} \pi a} \Re\left[e^{i \rho(a)} U\left(i a, x e^{-\pi i / 4}\right)\right], \\
& W(a,-x)=\sqrt{2 / k(a)} e^{\frac{1}{4} \pi a} \Im\left[e^{i \rho(a)} U\left(i a, x e^{-\pi i / 4}\right)\right] .
\end{aligned}
$$

Observe that Eq. (2.5) gives, up to a normalization factor, the normalized wave functions $\Phi(a, x)$ of Eq. (1.3), which are an orthonormal set in the sense that

$$
\int_{-\infty}^{\infty} \bar{\Phi}\left(a^{\prime}, x\right) \Phi(a, x) d x=\delta\left(a-a^{\prime}\right),
$$

with $\delta\left(a-a^{\prime}\right)$ the Dirac distribution. It is easy to check that $W(a, x)$ also satisfies orthogonality relations (see [?]).

\section{Approximations for moderate $a$ and $x$}

\subsection{Maclaurin series}

Maclaurin series are

$$
W(a, x)=W(a, 0) w_{1}(a, x)+W^{\prime}(a, 0) w_{2}(a, x),
$$


where $w_{1}(a, x)$ and $w_{2}(a, x)$ are the even and odd solutions of (1.6). The initial values are given in (2.1). We have

$$
w_{1}(a, x)=\sum_{n=0}^{\infty} \alpha_{n}(a) \frac{x^{2 n}}{(2 n) !}, \quad w_{2}(a, x)=\sum_{n=0}^{\infty} \beta_{n}(a) \frac{x^{2 n+1}}{(2 n+1) !},
$$

where $\alpha_{n}(a), \beta_{n}(a)$ satisfy the recursion

$$
\begin{aligned}
& \alpha_{n+2}=a \alpha_{n+1}-\frac{1}{2}(n+1)(2 n+1) \alpha_{n}, \\
& \beta_{n+2}=a \beta_{n+1}-\frac{1}{2}(n+1)(2 n+3) \beta_{n}, \\
& \alpha_{0}(a)=1, \quad \alpha_{1}(a)=a, \quad \beta_{0}(a)=1, \quad \beta_{1}(a)=a .
\end{aligned}
$$

The recursions in (3.3) can be modified to obtain recursions for

$$
a_{n}=\frac{\alpha_{n}(a)}{(2 n) !}, \quad b_{n}=\frac{\beta_{n}(a)}{(2 n+1) !} .
$$

We have

$$
\begin{aligned}
& a_{n+2}=\frac{a a_{n+1}-\frac{1}{4} a_{n}}{(2 n+3)(2 n+4)} \\
& b_{n+2}=\frac{a b_{n+1}-\frac{1}{4} b_{n}}{(2 n+4)(2 n+5)},
\end{aligned}
$$

with initial values

$$
a_{0}=1, \quad a_{1}=\frac{1}{2} a, \quad b_{0}=1, \quad b_{1}=\frac{1}{6} a .
$$

The computation of $W(a, \pm x)$ and their derivatives can be done in one run because the functions $w_{1}(a, x)$ and $w_{2}(a, x)$ are even and odd functions.

\subsubsection{The initial value $W(a, 0)$}

To obtain an algorithm for the initial values in (2.1) we can use an asymptotic expansion when $a$ is large. When $a$ is not large we choose an integer $N$, such that $N+\frac{1}{2} i a$ is large enough for using an asymptotic expansion. Afterwards we use recursion to obtain the wanted value of $W(a, 0)$.

We have (see [?, p. 67])

$$
\frac{\Gamma\left(\frac{1}{4}+z\right)}{\Gamma\left(\frac{3}{4}+z\right)} \sim \frac{1}{\sqrt{z}} \sum_{n=0}^{\infty} \frac{d_{n}}{z^{2 n}}
$$


where the first coefficients are given by

$$
\begin{aligned}
& d_{0}=1, \quad d_{1}=-\frac{1}{64}, \quad d_{2}=\frac{21}{8192}, \quad d_{3}=-\frac{671}{524288}, \\
& d_{4}=\frac{180323}{134217728}, \quad d_{5}=-\frac{20898423}{8589934592} .
\end{aligned}
$$

Writing $z=x+i y$ in polar coordinates $z=w e^{i \theta}$ we obtain an expansion of

$$
\frac{\Gamma\left(\frac{1}{4}+x+i y\right)}{\Gamma\left(\frac{3}{4}+x+i y\right)} \frac{\Gamma\left(\frac{1}{4}+x-i y\right)}{\Gamma\left(\frac{3}{4}+x-i y\right)} \sim \frac{1}{w} \sum_{n=0}^{\infty} \frac{g_{n}}{w^{2 n}}
$$

where

$$
\begin{aligned}
& g_{0}=1, \quad g_{1}=\frac{2 s^{2}-1}{32}, \quad g_{2}=\frac{84 s^{4}-84 s^{2}+11}{2048}, \\
& g_{3}=\left(2 s^{2}-1\right) \frac{2684 s^{4}-2684 s^{2}+173}{65536}, \\
& g_{4}=\frac{2885168 s^{8}-5770336 s^{6}+3609144 s^{4}-723976 s^{2}+22931}{8388608}, \\
& g_{5}=\left(2 s^{2}-1\right) \times \\
& \frac{334374768 s^{8}-668749536 s^{6}+397250360 s^{4}-62875592 s^{2}+1319183}{268435456},
\end{aligned}
$$

and $s=\sin \theta=y / \sqrt{x^{2}+y^{2}}$.

From this expansion, with $x=N, y=a / 2$, the requested value of $W(a, 0)$ can be obtained. We start from a large enough $N>0$ and then compute

$$
\left|\frac{\Gamma\left(\frac{1}{4}+\frac{1}{2} i a\right)}{\Gamma\left(\frac{3}{4}+\frac{1}{2} i a\right)}\right|=R_{N}\left|\frac{\Gamma\left(\frac{1}{4}+N+\frac{1}{2} i a\right)}{\Gamma\left(\frac{3}{4}+N+\frac{1}{2} i a\right)}\right| .
$$

$R_{0}=1$ and $R_{N}$ can be computed recursively by

$$
R_{n+1}=\sqrt{\frac{\left(\frac{3}{4}+n\right)^{2}+\frac{1}{4} a^{2}}{\left(\frac{1}{4}+n\right)^{2}+\frac{1}{4} a^{2}}} R_{n}, \quad n=0,1, \ldots, N-1 .
$$

\subsection{Chebyshev expansions}

The differential equation of the $W$-functions is given by (1.6), with even and odd solutions $w_{1}(a, x)$ and $w_{2}(a, x)$, as written in (3.2). We scale the 
independent variable by considering a new variable $z$ such that $x=2 \sqrt{\gamma} z$ for some $\gamma>0$. Then, we arrive at the equation

$$
\ddot{y}+4 \gamma\left(\gamma z^{2}-a\right) y=0, \quad \gamma>0,
$$

where dots mean derivatives with respect to $z$. The equation has the independent solutions

$$
v_{1}(a, z)=w_{1}(a, 2 \sqrt{\gamma} z), \quad v_{2}(a, z)=w_{2}(a, 2 \sqrt{\gamma} z) .
$$

We compute these solutions in terms of two Chebyshev series

$$
v_{i}(a, z)=\sum_{k=0}^{\infty}{ }^{\prime} c_{k} T_{k}(z), \quad-1 \leq z \leq 1,
$$

$i=1,2$, where the prime means that the $T_{0}(z)$ term has to be halved. Of course, $c_{2 j+1}=0, j \in \mathbb{N}$, for the case of $w_{1}$ and $c_{2 j}=0$ for the case of $w_{2}$.

The coefficients can be computed by plugging the expansions into the differential equation and using properties of Chebyshev polynomials. This is similar to Luke's approach for the Bessel function $J_{\nu}(x)$, see [?, p. 234] and [?, Chap. 2]. We denote

$$
\dot{v}_{i}(a, z)=\sum_{k=0}^{\infty}{ }^{\prime} c_{k}^{(1)} T_{k}(z), \quad \ddot{v}_{i}(a, z)=\sum_{k=0}^{\infty}{ }^{\prime} c_{k}^{(2)} T_{k}(z) .
$$

Now, substituting (3.15) and (3.16) and using [?, Eq. (3.84)] we get

$$
\begin{aligned}
& c_{k}^{(2)}+\frac{1}{4} A\left(c_{k+2}+2 c_{k}+c_{k-2}\right)+B c_{k}=0, \\
& A=4 \gamma^{2}, \quad B=-4 \gamma a .
\end{aligned}
$$

Next, shifting the index in (3.17) and using ([?, Eq. (3.80)])

$$
c_{k-1}^{(s+1)}-c_{k+1}^{(s+1)}=2 k c_{k}^{(s)} \quad s=0,1, \ldots, \quad i=1,2, \ldots,
$$

(where $c_{k}^{(0)}=c_{k}$ ) we arrive at

$$
2(k-1) c_{k-1}^{(1)}=\Delta_{k-1}=\frac{1}{4} A\left(c_{k+2}+c_{k}-c_{k-2}-c_{k-4}\right)+B\left(c_{k}-c_{k-2}\right) .
$$

Shifting the index in (3.19), using $2(k+1) c_{k+1}^{(1)}=\Delta_{k+1}$ and applying again (3.18), we obtain

$$
4 k\left(k^{2}-1\right) c_{k}=(1+k) \Delta_{k-1}+(1-k) \Delta_{k+1} .
$$


In this way, we obtain the following recurrence relation

$$
\begin{aligned}
& f_{4}(k) c_{k+4}+f_{2}(k) c_{k+2}+f_{0}(k) c_{k}+f_{-2}(k) c_{k-2}+f_{-4}(k) c_{k-4}=0 \\
& f_{4}(k)=(1-k), \quad f_{-4}(k)=-f_{4}(-k) \\
& f_{2}(k)=2\left(1-2 \frac{a(1-k)}{\gamma}\right), \quad f_{-2}(k)=-f_{2}(-k) \\
& f_{0}(k)=2 k\left(1-\frac{4 a}{\gamma}-2 \frac{k^{2}-1}{\gamma^{2}}\right)
\end{aligned}
$$

For determining the coefficients $c_{k}$, we can use this recurrence relation. Before doing this, it is crucial to determine the conditioning of the recurrence process, that is, the growth of the wanted solution.

For the moment, we concentrate on the coefficients $y_{k}=c_{2 k}$ (corresponding to the even solution). For the odd solutions the discussion is similar. For these coefficients, we introduce the recurrence relation

$$
\sum_{n=0}^{4} a_{k}^{(n)} y_{n+k}=0,
$$

where the coefficients have the following asymptotic behaviour:

$$
\begin{aligned}
& a_{k}^{(4)} \sim-2 k, \quad a_{k}^{(2)} \sim 8 k a / \gamma, \quad a_{k}^{(3)} \sim-32 k^{3} / \gamma^{2}, \\
& a_{k}^{(1)} \sim 8 k a / \gamma, \quad a_{k}^{(0)} \sim-2 k .
\end{aligned}
$$

Invoking the Perron-Kreuser theorem [?] (see [?] for a more recent description), we conclude that there exist two groups of solutions of the differential equation, one of them satisfying

$$
\limsup _{k \rightarrow+\infty} \sqrt[k]{\frac{\left|y_{k}\right|}{k !}}=\frac{4}{\gamma},
$$

and the second one

$$
\limsup _{k \rightarrow+\infty} \sqrt[k]{k !\left|y_{k}\right|}=\frac{\gamma}{4} .
$$

Each set is a linear space of dimension two generated by two independent solutions. 
The wanted solution lies in the second set. Clearly, this has to be the case because, for instance, taking into account that $T_{2 k}(0)=(-1)^{k}$, we have

$$
\sum_{k=0}^{\infty} '(-1)^{k} c_{2 k}=w_{1}(a, 0)=1 .
$$

and because the solutions of the set satisfying (3.24) are such that $\left|y_{k}\right| \rightarrow$ $+\infty$ as $k \rightarrow+\infty$, it is clear that the coefficients $y_{k}=c_{2 k}$ in (3.26) are not in this first set, but on the second set satisfying (3.25).

The second set of solutions is minimal with respect to the first set in the sense that, if $y_{k}^{(2)}$ is a solution of the second set and $y_{k}^{(1)}$ a solution of the first set, then, as a consequence of (3.24) and (3.25), $\lim _{k \rightarrow+\infty} y_{k}^{(2)} / y_{k}^{(1)}=0$. Minimal solutions can not be computed by using the recurrence relation in the forward direction; backward recursion is however possible.

Furthermore, in the case in which a $n$-term recurrence relation possesses a single minimal solution (up to constant multiplicative factors), the minimal solution can be computed by starting the recursion with arbitrary $y_{N+n}, \ldots$, $y_{N+1}$ (but not all zero) and using a normalization condition. This is Miller's algorithm for computing minimal solutions (see, for instance, [?, §4.6]).

In the present case, the set of minimal solutions has dimension two and we will need a second normalizing condition in order to determine the wanted minimal solution, as suggested in [?, pp. 163-164]).

For the even solution, we compute the coefficients $c_{2 k}$ starting with

$$
c_{2 N+6}=c_{2 N+4}=c_{2 N+2}=0, \quad c_{2 N}=\epsilon,
$$

with $\epsilon$ a small number in order to minimize possible overflows; of course, other selections of starting values are possible. We consider these starting values for two different values of $N$, say $N_{a}$ and $N_{b}<N_{a}$. This generates two minimal solutions. We normalize the solutions using (3.26), and we obtain two minimal solutions $c_{2 k}^{(a)}$ and $c_{2 k}^{(b)}$ such that

$$
\sum_{k=0}^{N_{a}}{ }^{\prime}(-1)^{k} c_{2 k}^{(a)}=1 \quad \text { and } \quad \sum_{k=0}^{N_{b}}{ }^{\prime}(-1)^{k} c_{2 k}^{(b)}=1 .
$$

Now, we consider a second normalization condition for building the wanted solution from a linear combination of $c_{2 k}^{(a)}$ and $c_{2 k}^{(b)}$. For this, we consider the relation, $C_{z^{2}}\left(v_{1}(a, z)\right)=C_{z^{2}}\left(w_{1}(a, 2 \sqrt{\gamma} z)\right)=2 a \gamma$ (see (3.2) and (3.3)), where $C_{z^{2}}(f(z))$ denotes the $z^{2}$ coefficient in the expansion of 
$f(z)$ in Maclaurin series. Then, we have, using the explicit polynomial representation for Chebyshev polynomials, [?, Eqs. (3.32-34)]

$$
2 a \gamma=\sum_{k=0}^{\infty}{ }^{\prime} c_{2 k} C_{z^{2}}\left(T_{2 k}(z)\right)=-2 \sum_{k=1}^{\infty}(-1)^{k} k^{2} c_{2 k} .
$$

Now, compute $S_{a}=\sum_{k=1}^{N_{a}}(-1)^{k} k^{2} c_{2 k}^{(a)}$ and $S_{b}=\sum_{k=1}^{N_{a}}(-1)^{k} k^{2} c_{2 k}^{(b)}$ and build the sequence

$$
c_{2 k}=\mu c_{2 k}^{(a)}+(1-\mu) c_{2 k}^{(b)}, \quad \mu=\left(S-S_{b}\right) /\left(S_{a}-S_{b}\right), \quad S=-a \gamma .
$$

The new sequence satisfies the normalizing conditions (3.26) and (3.29) approximately.

With this, one has an expansion

$$
w_{1}(a, 2 \sqrt{\gamma} z) \simeq \sum_{k=0}^{N_{a}}{ }^{\prime} c_{2 k} T_{2 k}(z), \quad z \in[-1,1],
$$

and particularizing for $z=1$

$$
w_{1}(a, 2 \sqrt{\gamma}) \simeq \sum_{k=0}^{N_{a}}{ }^{\prime} c_{2 k} .
$$

For the odd solution $v_{2}(a, z)=w_{2}(a, 2 \sqrt{\gamma} z)$ we follow a similar approach. The normalizing conditions consist in requiring that the coefficients $C_{z}\left(w_{2}(a, 2 \sqrt{\gamma} z)\right)$ and $C_{z^{3}}\left(w_{2}(a, 2 \sqrt{\gamma} z)\right)$ correspond to (3.2) and (3.3); they read

$$
\begin{aligned}
& \sum_{k=0}^{\infty}(-1)^{k}(k+1 / 2) c_{2 k+1}=\sqrt{\gamma} \\
& \sum_{k=0}^{\infty}(-1)^{k} k(k+1 / 2)(k+1) c_{2 k+1}=-a \gamma^{3 / 2} .
\end{aligned}
$$

With this the coefficients $c_{2 k+1}$ can be computed and, similarly as before,

$$
w_{2}(a, 2 \sqrt{\gamma} z) \simeq \sum_{k=0}^{N_{a}} c_{2 k+1} T_{2 k+1}(z)
$$

and particularizing for $z=1$ :

$$
w_{2}(a, 2 \sqrt{\gamma}) \simeq \sum_{k=0}^{N_{a}} c_{2 k+1} .
$$


For computing the derivatives, we consider the expansions (3.31) and (3.34). For instance, for the even part we have, taking the derivative,

$$
\dot{v}_{1}(a, z)=2 \sqrt{\gamma} w_{1}^{\prime}(a, x) \simeq \sum_{k=1}^{N_{a}} c_{2 k} \dot{T}_{2 k}(z), x=2 \sqrt{\gamma} z .
$$

Particularizing for $z=1$ and taking into account that $\dot{T}_{n}(1)=n^{2}$ we have

$$
w_{1}^{\prime}(a, 2 \sqrt{\gamma}) \simeq \frac{1}{2 \sqrt{\gamma}} \sum_{k=1}^{N_{a}} 4 k^{2} c_{2 k} .
$$

Similarly,

$$
w_{2}^{\prime}(a, 2 \sqrt{\gamma}) \simeq \frac{1}{2 \sqrt{\gamma}} \sum_{k=0}^{N_{a}}(2 k+1)^{2} c_{2 k+1} .
$$

An alternative but equivalent way of computing the derivatives is by considering

$$
2 \sqrt{\gamma} w_{1}^{\prime}(a, 2 \sqrt{\gamma} z)=\sum_{k=0}^{\infty} c_{2 k+1}^{(1)} T_{2 k+1}(z),
$$

where the coefficients $c_{2 k+1}^{(1)}$ can be computing recursively in parallel with the coefficients $c_{2 k}$ by means of (3.18). However, once the coefficients $c_{2 i}$ have been computed, it seems preferable to use (3.37) and (3.38).

For the numerical computation of the even and odd solutions with the method described, some precautions should be taken with the selection of the initial values $N_{a}$ and $N_{b}$ for starting the recursion process. Particularly, it should be noted that considering the starting values (3.27) does no guarantee that the generated solutions are "sufficiently different". In the method, it is crucial that we obtain two independent minimal solutions $c_{2 k}^{(a)}$ and $c_{2 k}^{(b)}$ such that the wanted solution can be computed as a linear combination in a numerically stable way. But this stability may accidentally fail. A way to eliminate these rare errors consists in running the method for two sets of values of $N_{a}$ and $N_{b}$ and to retain the solution which passes some numerical test. As test, the computation of the Wronskian relation

$$
w_{1}(a, x) w_{2}^{\prime}(a, x)-w_{1}^{\prime}(a, x) w_{2}(a, x)=1
$$

can be considered. An a priori estimation of the values of $N_{a}$ and $N_{b}$ is a difficult task and depends on the accuracy needed and on the region of application of the method; some numerical experiments can be considered 
for this task. For the range of application of this method (see $\S 5$ ), values close to 100 are enough for determining the coefficients with sufficient accuracy. Specifically, the two sets of values considered in $\S 5$ are $N_{a}=110, N_{b}=100$ and $N_{a}=108, N_{b}=90$; for each $a$ and $x$, the set which gives a smaller deviation from Eq. (3.40) is chosen.

As we will see in $\S 5$, Chebyshev expansions are valid for a larger $x$ range than Maclaurin series, but the same can not be said with respect to the $a$ parameter. Chebyshev expansions have the interesting feature that, once the coefficients $c_{k}$ for a given $a$ have been computed, the functions $w_{1}(a, 2 \sqrt{\gamma} z)$ and $w_{1}(a, 2 \sqrt{\gamma} z)$ can be efficiently evaluated for any $z \in[-1,1]$ by using Clenshaw's method (see, for instance, [?, §3.7.1]). In our previous discussion, we have fixed the value $z=1$ and let $\gamma$ vary for simplicity and in order to study the range of validity of the method (see $\S 5$ ).

\subsection{Local Taylor series}

In $\S 3.1$, we considered Maclaurin series for computing $W(a, \pm x)$ close to $x=0$. It is possible to consider successive local Taylor series for computing the functions further away from $x=0$, giving a number of values of the function at some values $x_{i}$ of $x$, with $x_{0}<x_{1}<\cdots<x_{n}$.

Consider, for instance, $x_{0}=0, x_{n}>0$ and that we aim at computing $y(x)=W(a, x)($ or $y(x)=W(a,-x))$ and its derivative at $x=x_{i}, i=$ $0, \ldots, n$. For simplicity, let us consider a constant step size $h=x_{i+1}-x_{i}$.

Assuming that $y(0)$ and $y^{\prime}(0)$ are available (see $\S 3.1 .1$ ), the idea is to compute $y\left(x_{1}\right)=y\left(x_{0}+h\right)$ using Taylor series for $y(x)$ around $x_{0}$ and proceed similarly for the remaining nodes $x_{i}$. That is, we compute

$$
\begin{aligned}
& y\left(x_{i+1}\right)=\sum_{k=0}^{N} y^{(k)}\left(x_{i}\right) \frac{h^{k}}{k !}+\mathcal{O}\left(h^{N+1}\right), \\
& y^{\prime}\left(x_{i+1}\right)=\sum_{k=0}^{N} y^{(k+1)}\left(x_{i}\right) \frac{h^{k}}{k !}+\mathcal{O}\left(h^{N+1}\right) .
\end{aligned}
$$

Similarly, one can compute the function at $x_{i}$ from the values at $x_{i+1}$, simply by interchanging the roles of $x_{i}$ and $x_{i+1}$ and the sign of $h$.

Let us observe that the Maclaurin series of $\S 3.1$ are the particular case $i=0, x_{0}=0$ in Eq. (3.41); therefore, the next discussion gives an alternative method for computing the Maclaurin series.

The successive derivatives $y^{(k)}$ can be computed by differentiation of the 
differential equation. We have, differentiating $k$ times, $k \geq 2$ :

$$
y^{(k+2)}+\left(\frac{1}{4} x^{2}-a\right) y^{(k)}+\frac{1}{2} x k y^{(k-1)}+\frac{1}{4} k(k-1) y^{(k-2)}=0,
$$

which allows computing derivatives at $x=x_{i}$ when $y^{(j)}\left(x_{i}\right), j=0,1,2,3$ are known. $y^{(0)}\left(x_{i}\right)$ and $y^{(1)}\left(x_{i}\right)$ are known from the previous step, and

$$
\begin{aligned}
& y^{(2)}\left(x_{i}\right)=-\left(\frac{1}{4} x_{i}^{2}-a\right) y^{(0)}\left(x_{i}\right), \\
& y^{(3)}\left(x_{i}\right)=-\left(\frac{1}{4} x_{i}^{2}-a\right) y^{(1)}\left(x_{i}\right)-\frac{1}{2} x_{i} y^{(0)}\left(x_{i}\right) .
\end{aligned}
$$

For computing the successive derivatives, it is necessary that the recursion process for Eq. (3.42) is well conditioned. Considering the PerronKreuser theorem [?], it is easy to see that all the solutions of (3.42) increase as $k \rightarrow+\infty$ in such a way that

$$
\limsup _{k \rightarrow+\infty} \sqrt[k]{\frac{\left|y^{(k)}\right|}{(k !)^{1 / 2}}}=2^{-1 / 2},
$$

and that, hence, one can not expect that solutions exist which become negligible with respect to some other solutions of the same recurrence relation as $k \rightarrow+\infty$. Forward computation, therefore, is not bad conditioned as $k$ becomes large, because the successive derivatives of $W(a, x)$ (or $W(a,-x)$ ) will not become negligible.

Because $W(a, x)$ and $W(a,-x)$ are entire functions and because the derivatives can be computed in a stable way, one expects that, provided that the computation of the sums (3.41) is numerically stable, the process of generating values of $W(a, x)(W(a,-x))$ and its derivative at $x_{0}<x_{1}<$ $\ldots<x_{n}$ is a stable numerical process.

However, for ensuring numerical stability of the sums, the direction of application of the Taylor series method, or in other words, the sign of the step $h$, should be chosen with care, depending on the growth of the solutions.

\subsubsection{Conditioning of the integration direction for Taylor series}

It is crucial to analyze the relative size of the solutions of the differential equation $W(a, x)$ and $W(a,-x)$ in order to determine stable directions of integration. 
Consider the functions $\left.M_{1}(a, x)=\left[\left(\frac{1}{2} x W(a, x)\right)^{2}+W^{\prime}(a, x)^{2}\right)\right]^{1 / 2}$ and $M_{2}(a, x)=\left[\left(\frac{1}{2} W(a,-x)\right)^{2}+W^{\prime}(a,-x)^{2}\right]^{1 / 2}$. Then, considering the asymptotic approximations for large $x$ (4.1) one sees at once that

$$
\lim _{x \rightarrow+\infty} \frac{M_{2}(a, x)}{M_{1}(a, x)}=\sqrt{1+e^{\pi a}}+e^{\pi a} .
$$

Therefore, for negative $a, M_{1}(a, x)$ is of size comparable to $M_{2}(a, x)$ as $x \rightarrow+\infty$. However, for positive $a, M_{1}(a, x)$ becomes much smaller than $M_{2}(a, x)$, much more as $a$ increases (but $\left.x^{2}>>4|a|\right)$. Notice that $M_{1}(a, 0)=$ $M_{2}(a, 0)$; therefore $W(a, x)$ decreases steeply in relation with $W(a,-x)$ as $x$ grows, and the same is true for the derivatives.

With this, it is clear that when $a>0$ integration of the differential equation with Taylor series for $W(a,-x)$ and $\frac{d}{d x} W(a,-x)$ can be done in the direction of increasing $x$, because they dominate over $W(a, x)$ and $W^{\prime}(a, x)$. However, this process is badly conditioned for $W(a, x)$ and $W^{\prime}(a, x)$, and the integration must be done in the opposite direction in order to recover a well conditioned process.

Therefore, for computing $W(a, x), W(a,-x)$ and its derivatives with local Taylor series, the following strategies should be considered:

1. For $W(a,-x)$ and $\frac{d}{d x} W(a,-x)$ : compute Taylor series in increasing order of the nodes $x_{0}<x_{1}<\cdots$, starting with $x_{0}=0$ (see $\S 3.1 .1$ for the values of $\left.W(a, 0), W^{\prime}(a, 0)\right)$.

2. For $W(a, x)$ and $W^{\prime}(a, x)$ :

(a) If $a<0$ proceed as before for $W(a,-x)$ and its derivative.

(b) If $a>0$, integrate in the direction of decreasing $x$, in the order $x_{0}>x_{1}>\cdots$ taking a negative step size $h=x_{i+1}-x_{i}$. For large enough $x_{0}$, starting values are provided by the asymptotic expansions of Eqs. (4.27) to (4.30).

Using this scheme, and considering moderately small values of $h$ (for instance $|h|=0.05)$ a considerably wide region of parameters can be covered. For instance, we have checked that a relative accuracy better than $510^{-14}$ is attained in the $(a, x)$-region $[-50,50] \times[0,50]$. Considering $N=20$ in (3.41) is enough for this step size. 


\section{Asymptotic expansions}

$W(a, x)$ is oscillating when $x^{2}>4 a$. For large enough $x$ we can use the Poincaré-type asymptotic expansions [?, Eqs. 19.21.2-7]:

$$
\begin{aligned}
& W(a, x)=\sqrt{2 k(a) / x}\left(s_{1}(a, x) \cos \chi-s_{2}(a, x) \sin \chi\right), \\
& W(a,-x)=\sqrt{2 /(k(a) x)}\left(s_{1}(a, x) \sin \chi+s_{2}(a, x) \cos \chi\right),
\end{aligned}
$$

where $\chi=\frac{1}{4} x^{2}-a \ln x+\frac{1}{4} \pi+\frac{1}{2} \phi_{2}(a), k(a)$ is given in (2.6) and $\phi_{2}(a)$ in (2.7). The functions $s_{j}(a, x)$ have the asymptotic expansions

$$
\begin{aligned}
& s_{1}(a, x) \sim 1+\frac{v_{2}}{1 ! 2 x^{2}}-\frac{u_{4}}{2 ! 2^{2} x^{4}}-\frac{v_{6}}{3 ! 2^{3} x^{6}}+\frac{u_{8}}{4 ! 2^{4} x^{8}}+\ldots, \\
& s_{2}(a, x) \sim-\frac{u_{2}}{1 ! 2 x^{2}}-\frac{v_{4}}{2 ! 2^{2} x^{4}}+\frac{u_{6}}{3 ! 2^{3} x^{6}}+\frac{v_{8}}{4 ! 2^{4} x^{8}}-\ldots
\end{aligned}
$$

where $u_{r}+i v_{r}=\Gamma\left(r+\frac{1}{2}+i a\right) / \Gamma\left(\frac{1}{2}+i a\right)$. These coefficients follow also from the recursion

$$
\left\{\begin{array}{l}
u_{r+1}=\left(r+\frac{1}{2}\right) u_{r}-a v_{r}, \\
v_{r+1}=\left(r+\frac{1}{2}\right) v_{r}+a u_{r},
\end{array}\right.
$$

with initial values $u_{0}=1, v_{0}=0$.

These expansions are rather simple (the only non-trivial element is the function $\phi_{2}(a)$, defined in Eq. (2.7)), but we prefer expansions that are described next (§4.1.2), because these are valid when $|x|+|a|$ is large and $x^{2} / 4-a>0$. Also, we are giving expansions for the monotonic region $\left(x^{2} / 4-a<0\right)$ when $a$ is large and positive $(\S 4.1 .1)$ and Airy type expansions for the transition region $(\S 4.2)$.

\subsection{Expansions in terms of elementary functions}

We use the notation [?]

$$
a=\frac{1}{2} \mu^{2}, \quad x=\mu t \sqrt{2} .
$$

In the new variables $t$ and $\mu$ the differential equation (1.6) reads

$$
\frac{d^{2} w}{d t^{2}}+\mu^{4}\left(t^{2}-1\right) w=0 .
$$


We consider two cases: $-1+\delta \leq t \leq 1-\delta$ (monotonic region) and $t \geq 1+\delta$ (oscillatory region), where $\delta$ is a small positive number. Initially, we assume that $a>0$ although the expansion in the region $t \geq 1+\delta$ will be also valid for $a<0$ (and for $a=0$ if $x$ is large enough) after undoing the change of variables and restoring the formulas to the $a$ and $x$ variables.

\subsubsection{The case $-1+\delta \leq t \leq 1-\delta$}

From [?, (12.15)] we have

$$
\begin{aligned}
& W(a, x) \sim \frac{\ell(\mu) e^{\mu^{2} \eta(t)-\frac{1}{4} \pi \mu^{2}}}{\sqrt{2}\left(1-t^{2}\right)^{\frac{1}{4}}} \sum_{s=0}^{\infty} \frac{(-1)^{s} u_{s}(t)}{\left(1-t^{2}\right)^{\frac{3}{2} s} \mu^{2 s}}, \\
& W^{\prime}(a, x) \sim-\frac{1}{2} \mu \ell(\mu) e^{\mu^{2} \eta(t)-\frac{1}{4} \pi \mu^{2}}\left(1-t^{2}\right)^{\frac{1}{4}} \sum_{s=0}^{\infty} \frac{(-1)^{s} v_{s}(t)}{\left(1-t^{2}\right)^{\frac{3}{2} s} \mu^{2 s}},
\end{aligned}
$$

where

$$
\eta(t)=\frac{1}{2} \arccos t-\frac{1}{2} t \sqrt{1-t^{2}} .
$$

We can also write

$$
\mu^{2} \eta(t)-\frac{1}{4} \pi \mu^{2}=-a\left(\arcsin t+t \sqrt{1-t^{2}}\right),
$$

showing that the argument in the exponential functions in (4.6) is an odd function of $t$.

Details on the coefficients $u_{s}(t)$ and $v_{s}(t)$ can be found in [?] and [?, $\S 2.1]$. The quantity $\ell(\mu)$ in (4.6) has the asymptotic expansion [?, 12.11]

$$
\ell(\mu) \sim \frac{2^{\frac{1}{4}}}{\mu^{\frac{1}{2}}} \sum_{s=0}^{\infty} \frac{\ell_{s}}{\mu^{4 s}},
$$

where the first five coefficients are

$$
\begin{aligned}
& \ell_{0}=1, \quad \ell_{1}=-\frac{1}{1152}, \quad \ell_{2}=-\frac{16123}{39813120}, \\
& \ell_{3}=-\frac{2695447331}{4815794995200}, \quad \ell_{4}=-\frac{37598748996091}{2219118333788} .
\end{aligned}
$$

The numbers $\ell_{0}, \ell_{1}, \ell_{2}$ are given by Olver, but for a numerical algorithm we needed a few extra ones. To evaluate more coefficients we use [?, 12.512.12] and see that they are related to coefficients $\gamma_{s}$ occurring in

$$
\Gamma\left(\frac{1}{2}+z\right) \sim \sqrt{2 \pi} e^{-z} z^{z} \sum_{k=0}^{\infty} \frac{\gamma_{s}}{z^{s}}, \quad z \rightarrow \infty .
$$


The coefficients $\gamma_{s}$ can be obtained by using standard methods for the gamma function. We have

$$
\begin{aligned}
& \gamma_{0}=1, \quad \gamma_{1}=-\frac{1}{24}, \quad \gamma_{2}=\frac{1}{1152}, \quad \gamma_{3}=\frac{1003}{414720} \\
& \gamma_{4}=-\frac{4027}{39813120}, \quad \gamma_{5}=-\frac{5128423}{6688604160}, \quad \gamma_{6}=\frac{168359651}{4815794995200} \\
& \gamma_{7}=\frac{68168266699}{115579079884800} .
\end{aligned}
$$

Next we introduce the modified expansion

$$
M(\mu) \sim \sum_{s=0}^{\infty} \frac{\gamma_{s}}{\left(\frac{1}{2} i \mu^{2}\right)^{s}},
$$

and from Olver's results it follows that the coefficients $\ell_{s}$ in (4.9) occur in the asymptotic relation

$$
\ell(\mu)=\frac{2^{\frac{1}{4}}}{\mu^{\frac{1}{2}}} \frac{1+M(\mu)}{2 \sqrt{M(\mu)}} \sim \frac{2^{\frac{1}{4}}}{\mu^{\frac{1}{2}}} \sum_{s=0}^{\infty} \frac{\ell_{s}}{\mu^{4 s}} .
$$

\subsubsection{The case $t \geq 1+\delta$}

The relevant expansions for $U$ and $V$ and their derivatives are (see [?, $§ 2.1]$ )

$$
\begin{gathered}
U\left(-\frac{1}{2} \mu^{2}, \mu t \sqrt{2}\right)=\frac{h(\mu) e^{-\mu^{2} \xi}}{\left(t^{2}-1\right)^{\frac{1}{4}}} F_{\mu}(t), \\
U^{\prime}\left(-\frac{1}{2} \mu^{2}, \mu t \sqrt{2}\right)=-\frac{\mu}{\sqrt{2}} h(\mu)\left(t^{2}-1\right)^{\frac{1}{4}} e^{-\mu^{2} \xi} G_{\mu}(t), \\
V\left(-\frac{1}{2} \mu^{2}, \mu t \sqrt{2}\right)=\frac{e^{\mu^{2} \xi}}{\mu \sqrt{\pi} h(\mu)\left(t^{2}-1\right)^{\frac{1}{4}}} P_{\mu}(t), \\
V^{\prime}\left(-\frac{1}{2} \mu^{2}, \mu t \sqrt{2}\right)=\frac{\left(t^{2}-1\right)^{\frac{1}{4}} e^{\mu^{2} \xi}}{\sqrt{2 \pi} h(\mu)} Q_{\mu}(t),
\end{gathered}
$$

with expansions

$$
F_{\mu}(t) \sim \sum_{s=0}^{\infty} \frac{\phi_{s}(\tau)}{\mu^{2 s}}, \quad G_{\mu}(t) \sim \sum_{s=0}^{\infty} \frac{\psi_{s}(\tau)}{\mu^{2 s}},
$$




$$
P_{\mu}(t) \sim \sum_{s=0}^{\infty}(-1)^{s} \frac{\phi_{s}(\tau)}{\mu^{2 s}}, \quad Q_{\mu}(t) \sim \sum_{s=0}^{\infty}(-1)^{s} \frac{\psi_{s}(\tau)}{\mu^{2 s}},
$$

which are valid for $\mu+t \rightarrow \infty, \mu \geq 0, t \geq t_{0}$, for a fixed $t_{0}>1$. We have

$$
\begin{gathered}
h(\mu)=2^{-\frac{1}{4} \mu^{2}-\frac{1}{4}} e^{-\frac{1}{4} \mu^{2}} \mu^{\frac{1}{2} \mu^{2}-\frac{1}{2}}=2^{-\frac{1}{2}} a^{\frac{1}{2}-\frac{1}{4}} e^{-\frac{1}{2} a}, \\
\xi(t)=\frac{1}{2} t \sqrt{t^{2}-1}-\frac{1}{2} \ln \left(t+\sqrt{t^{2}-1}\right), \\
\tau=\frac{1}{2}\left(\frac{t}{\sqrt{t^{2}-1}}-1\right)=\frac{1}{2 \sqrt{t^{2}-1}\left(t+\sqrt{t^{2}-1}\right)},
\end{gathered}
$$

For obtaining real asymptotic expansions for the real functions $W(a, \pm x)$, we apply (2.9) and replace $\mu$ by $\mu e^{-\frac{1}{4} \pi i}$. This gives for $h(\mu)$ (see (4.21))

$$
h\left(\mu e^{-\frac{1}{4} \pi i}\right)=2^{-\frac{1}{4}} \mu^{-\frac{1}{2}} e^{-\frac{1}{4} \pi a} e^{i \sigma}, \quad \sigma=\frac{1}{8} \pi+\frac{1}{2} a-\frac{1}{2} a \ln a .
$$

Next, we write $F_{\mu \exp (-i \pi / 4)}(\tau)=F_{r}+i F_{i}$ and $G_{\mu \exp (-i \pi / 4)}(\tau)=G_{r}+i G_{i}$, and from (4.19) we have the expansions

$$
\begin{aligned}
& F_{r} \sim \sum_{s=0}^{\infty} \frac{(-1)^{s} \phi_{2 s}(\tau)}{(2 a)^{2 s}}, \quad F_{i} \sim \sum_{s=0}^{\infty} \frac{(-1)^{s} \phi_{2 s+1}(\tau)}{(2 a)^{2 s+1}}, \\
& G_{r} \sim \sum_{s=0}^{\infty} \frac{(-1)^{s} \psi_{2 s}(\tau)}{(2 a)^{2 s}}, \quad G_{i} \sim \sum_{s=0}^{\infty} \frac{(-1)^{s} \psi_{2 s+1}(\tau)}{(2 a)^{2 s+1}}
\end{aligned}
$$

In this way we obtain the representations for the $W$-functions

$$
\begin{gathered}
W(a, x)=\frac{\sqrt{k(a)}}{a^{\frac{1}{4}}\left(t^{2}-1\right)^{\frac{1}{4}}}\left(\cos \chi F_{r}-\sin \chi F_{i}\right), \\
W^{\prime}(a, x)=-\sqrt{k(a)} a^{\frac{1}{4}}\left(t^{2}-1\right)^{\frac{1}{4}}\left(\sin \chi G_{r}+\cos \chi G_{i}\right), \\
W(a,-x)=\frac{1}{\sqrt{k(a)} a^{\frac{1}{4}}\left(t^{2}-1\right)^{\frac{1}{4}}}\left(\sin \chi F_{r}+\cos \chi F_{i}\right), \\
W^{\prime}(a,-x)=-\frac{a^{\frac{1}{4}}\left(t^{2}-1\right)^{\frac{1}{4}}}{\sqrt{k(a)}}\left(\cos \chi G_{r}-\sin \chi G_{i}\right) .
\end{gathered}
$$

Here,

$$
\chi=\rho^{*}(a)+\frac{1}{4} \pi+2 a \xi(t) .
$$


where $\rho^{*}(a)$ is defined in (2.8). For large $a$ we have the asymptotic expansion

$$
\rho^{*}(a) \sim \frac{1}{4} a \ln \left(1+\frac{1}{4 a^{2}}\right)-\frac{1}{2 a} \sum_{k=0}^{\infty} \frac{d_{k}}{a^{2 k}},
$$

as $a \rightarrow \pm \infty$ where the coefficients $d_{k}$ can be expressed in terms of Bernoulli polynomials (for details, we refer to [?]). For computations it is convenient to expand also the logarithm in (4.32). This gives

$$
\rho^{*}(a) \sim \frac{1}{a} \sum_{k=0}^{\infty} \frac{\rho_{k}}{a^{2 k}}
$$

where the first five coefficients are:

$$
\rho_{0}=\frac{1}{48}, \quad \rho_{1}=\frac{7}{5760}, \quad \rho_{2}=\frac{31}{80640}, \quad \rho_{3}=\frac{127}{430080}, \quad \rho_{4}=\frac{511}{1216512} .
$$

For smaller $a$ one can compute $\rho(a)$ from and (2.7) and (2.8) by direct evaluation of $\Gamma(1 / 2+i a)$. Similarly as discussed in $\S 3.1 .1$, one can compute $\Gamma(N+1 / 2+i a)$ for large enough $N$ using asymptotics (Eq. (4.11) for $z=N+i a)$, and use recurrence in order to go down to $N=0$.

In terms of $x$ and $a$ :

$$
a^{\frac{1}{4}}\left(t^{2}-1\right)^{\frac{1}{4}}=\left(\frac{1}{4} x^{2}-a\right)^{\frac{1}{4}}
$$

and

$$
t=\frac{x}{2 \sqrt{a}}, \quad \tau=\frac{2 a}{V}, \quad V=4 \sqrt{\frac{1}{4} x^{2}-a}\left(\frac{1}{2} x+\sqrt{\frac{1}{4} x^{2}-a}\right) .
$$

The coefficients $\phi_{s}(\tau)$ and $\psi_{s}(\tau)$ are polynomials in $\tau$, and can be computed by recursion; see [?, §2.1.1].

To define the terms of the asymptotic expansion for $a=0$ we can scale the terms by writing

$$
\frac{\phi_{s}(\tau)}{(2 a)^{s}}=\frac{1}{V^{s}} p_{s}(\tau), \quad \frac{\psi_{s}(\tau)}{(2 a)^{s}}=\frac{1}{V^{s}} q_{s}(\tau),
$$

where $p_{s}(\tau)$ and $q_{s}(\tau)$ are polynomials of degree $2 s$. For example,

$$
\frac{\phi_{1}(\tau)}{2 a}=-\frac{1}{12 V}\left(20 \tau^{2}+30 \tau+9\right), \quad \frac{\psi_{1}(\tau)}{2 a}=\frac{1}{12 V}\left(28 \tau^{2}+42 \tau+15\right) .
$$


For numerical applications we need $t$-values strictly larger than unity. However, when $x$ and $a$ are large we have noticed that $t$ may be rather close to one.

From the Wronskian relation (2.2) it follows that the expansions (4.25) and (4.26) satisfy

$$
F_{r} G_{r}+F_{i} G_{i}=1 .
$$

We have verified that for a given $a \geq 0$ and $x \geq a / 10+15$ the value of $\left|F_{r} G_{r}+F_{i} G_{i}-1\right|$ (see the Wronskian (4.39)) is about $10^{-15}$ or less. For $x=a / 10+15$, the minimal value of $t$ is $1.22 \ldots$, which is assumed at $a=150$.

\subsection{Expansions in terms of Airy functions}

The representations to be given describe the asymptotic behaviour of $W(a, x)$ near the transition points of the equation (1.6) $x= \pm 2 \sqrt{a}$, or of (4.5) at $t= \pm 1$.

The following representations in terms of Airy functions are given in [?]:

$$
\begin{gathered}
W(a, x) \sim \frac{\pi^{\frac{1}{2}} \mu^{\frac{1}{3}} \ell(\mu) \phi(\zeta)}{2^{\frac{1}{2}} e^{\frac{1}{2} \pi a}}\left(\operatorname{Bi}(-z) A_{\mu}(\zeta)+\frac{\operatorname{Bi}^{\prime}(-z)}{\mu^{\frac{8}{3}}} B_{\mu}(\zeta)\right), \\
W^{\prime}(a, x) \sim-\frac{\pi^{\frac{1}{2}} \mu^{\frac{2}{3}} \ell(\mu)}{2 e^{\frac{1}{2} \pi a} \phi(\zeta)}\left(-\frac{\operatorname{Bi}(-z)}{\mu^{\frac{4}{3}}} C_{\mu}(\zeta)+\operatorname{Bi}^{\prime}(-z) D_{\mu}(\zeta)\right), \\
W(a,-x) \sim \frac{\pi^{\frac{1}{2}} \mu^{\frac{1}{3}} \ell(\mu) \phi(\zeta)}{2^{-\frac{1}{2}} e^{-\frac{1}{2} \pi a}}\left(\operatorname{Ai}(-z) A_{\mu}(\zeta)+\frac{\operatorname{Ai}^{\prime}(-z)}{\mu^{\frac{8}{3}}} B_{\mu}(\zeta)\right), \\
W^{\prime}(a,-x) \sim \frac{\pi^{\frac{1}{2}} \mu^{\frac{2}{3}} \ell(\mu)}{e^{-\frac{1}{2} \pi a} \phi(\zeta)}\left(-\frac{\operatorname{Ai}(-z)}{\mu^{\frac{4}{3}}} C_{\mu}(\zeta)+\operatorname{Ai}^{\prime}(-z) D_{\mu}(\zeta)\right),
\end{gathered}
$$

where $\ell(\mu)$ is given by the expansion in (4.9), $z=\mu^{\frac{4}{3}} \zeta$, and for $\zeta$ we have the relation

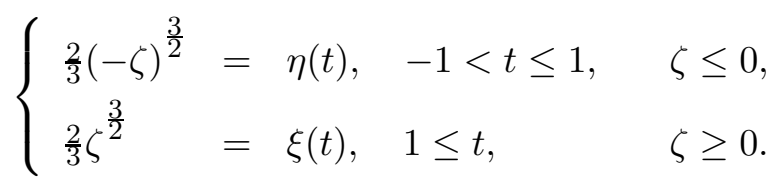

The function $\eta(t)$ is given in (4.7) and $\xi(t)$ in $(4.22) ; \zeta(t)$ is real for $t>-1$ and analytic at $t=1$. We can invert $\zeta(t)$ into $t(\zeta)$, and obtain

$$
t=1+2^{-1 / 3} \zeta-\frac{1}{10} 2^{-2 / 3} \zeta^{2}+\frac{11}{700} \zeta^{3}+\ldots .
$$


The function $\phi(\zeta)$ is given by

$$
\phi(\zeta)=\left(\frac{\zeta}{t^{2}-1}\right)^{\frac{1}{4}} .
$$

The functions $A_{\mu}(\zeta), B_{\mu}(\zeta), C_{\mu}(\zeta)$, and $D_{\mu}(\zeta)$ have asymptotic expansions of the form

$$
\begin{array}{ll}
A_{\mu}(\zeta) \sim \sum_{s=0}^{\infty}(-1)^{s} \frac{a_{s}(\zeta)}{\mu^{4 s}}, & B_{\mu}(\zeta) \sim \sum_{s=0}^{\infty}(-1)^{s} \frac{b_{s}(\zeta)}{\mu^{4 s}} \\
C_{\mu}(\zeta) \sim \sum_{s=0}^{\infty}(-1)^{s} \frac{c_{s}(\zeta)}{\mu^{4 s}}, & D_{\mu}(\zeta) \sim \sum_{s=0}^{\infty}(-1)^{s} \frac{d_{s}(\zeta)}{\mu^{4 s}} .
\end{array}
$$

Details on the coefficients can be found in [?] and [?, §3.1]. Let

$$
\chi(\zeta)=\frac{\phi^{\prime}(\zeta)}{\phi(\zeta)}
$$

Then

$$
\begin{aligned}
& C_{\mu}(\zeta)=\chi(\zeta) A_{\mu}(\zeta)+\zeta B_{\mu}(\zeta)+A_{\mu}^{\prime}(\zeta) \\
& D_{\mu}(\zeta)=A_{\mu}(\zeta)-\frac{1}{\mu^{4}}\left(\chi(\zeta) B_{\mu}(\zeta)+B_{\mu}^{\prime}(\zeta)\right)
\end{aligned}
$$

It follows that the coefficients $c_{s}(\zeta)$ and $d_{s}(\zeta)$ in (4.47) are given by

$$
\begin{aligned}
& c_{s}(\zeta)=\chi(\zeta) a_{s}(\zeta)+a_{s}^{\prime}(\zeta)+\zeta b_{s}(\zeta), \\
& d_{s}(\zeta)=a_{s}(\zeta)+\chi(\zeta) b_{s-1}(\zeta)+b_{s-1}^{\prime}(\zeta) .
\end{aligned}
$$

Several algorithms exist for the computation of Airy functions, both for real $[?, ?]$ and complex arguments [?, ?].

\section{$5 \quad$ Numerical tests}

Several numerical tests have been performed in order to show that the described methods are enough to cover a wide range of parameters.

The values are checked with the Wronskian relation (2.3). The methods have been intensively tested in the region $(x, a) \in[0,1000] \times[-100,100]$. Also, values of the functions are tested by comparing the results of different methods, when they are available for a same region and by comparing with function values computed by Maple, with a number of digits as large as 
needed. Comparison against Maple is considered in a more restricted region because the computation for large parameters becomes very slow, if not impossible at all (see the discussion on timings at the end of this section).

For comparing function values, it should be noted that when the functions become oscillatory, relative accuracy unavoidably worsens close to the zeros. For this reason, when $x$ is not small, it is convenient to consider the following auxiliary functions

$$
\begin{aligned}
& y_{1}(a, x)=\frac{1}{2} x W(a, x) \quad y_{2}(x)=W^{\prime}(a, x), \\
& M(a, x)=\left(y_{1}(a, x)^{2}+y_{2}(a, x)^{2}\right)^{1 / 2},
\end{aligned}
$$

and similarly for $W(a,-x)$ and $W^{\prime}(a,-x) . y_{1}$ and $y_{2}$ are oscillatory functions for large $x$ and of similar amplitude. In fact, considering (4.1) one sees that

$$
y_{1}(a, x) / M(a, x)=\cos \chi+o(1), \quad y_{2}(a, x) / M(a, x)=-\sin \chi+o(1),
$$

as $x \rightarrow+\infty$.

$M(a, x)$ can be interpreted as a modulus function, which is free of zeros. This is a good quantity for checking relative accuracy.

In order to determine the region of applicability of each method, we have compared Maclaurin, Chebyshev and Taylor series and asymptotic expansions (Airy-type and in terms of elementary functions) with the results provided by Maple (with 50 digits).

Figure 2 shows the comparison of the different methods against Maple for the computation of the function $M(a, x)(5.1)$ and for a relative accuracy better than $5 \times 10^{-13}$. The points where the claimed relative accuracy is not reached are shown.

Figures 4 and 3 show the regions of applicability of the methods in the $(x, a)$-plane for the computation of the $W(a, x)$ function and for an accuracy better than $5 \times 10^{-13}$. The selection of one method or another when alternatives are available in a same region, has been made depending on the efficiency of each of these methods.

The fitted curves separating the regions in the $(a, x)$-plane are explicitly given by: 
Figure 2: Computation of the $W(a, x)$ function: comparison against Maple of series (upper figure, left), Chebyshev expansions (upper figure, right), expansions in terms of elementary functions (lower figure, left) and Airy expansions (lower figure, right). The points where a $5 \times 10^{-13}$ relative accuracy is not reached, are shown. 


$$
\begin{aligned}
& f_{1}: a=7(x-2)^{2}+2.5, \\
& f_{2}: x=2, \\
& f_{3}: a=1.1 x^{2}+30.5, \\
& f_{4}: a=0.2(x-12)^{2}+30, \\
& f_{5}: a=0.75(x-12)^{2}, \\
& f_{6}: a=0.18 x^{2}-25, \\
& f_{7}: a=-2(x-4.8)^{2}-3, \\
& f_{8}: a=-5 \\
& f_{9}: x=5 \\
& f_{10}: x=12 .
\end{aligned}
$$

In order to check the validity of the fitted curves, an extensive test for the Wronskian has been performed. We have considered $10^{7}$ random points in the region of the $(x, a)$-plane $[0,1000] \times[-100,100]$ and the maximum relative deviation from 1 of the relation (2.3) was $4.810^{-13}$, consistent with our accuracy claim $\left(5 \times 10^{-13}\right)$.

The methods presented in this paper are much more efficient that the complex variable computations that are available in Maple (or Matlab). Next, we provide some examples comparing the performance of a Fortran 90 program using our methods against the computation of $W(a, x)$ via the relation (2.9) and using Maple10 with 16 digits. The timings for the Maple and Fortran programs are made in a same machine (a modern laptop).

For moderately large values of the parameters, when asymptotic expansions are used in the Fortran program, the advantage with respect to Maple is huge. For instance, Maple10 is around $10^{8}$ times slower for computing $W(50,80)$ than the Fortran program (which computes $W(a, x), W(a,-x)$ and their first derivatives): Maple takes $69.7 \mathrm{~s}$ and the Fortran program $6.410^{-6} \mathrm{~s}$. For larger values of the variables, the advantage of asymptotics with respect to Maple becomes even larger. The timing comparison for moderate values of the parameters is also favourable for our methods (but not so overwhelmingly better). For instance, for $x=10, a=20$ Maple takes 0.34 seconds to compute $W(a, x)$ while the local Taylor series take 0.002 seconds to compute $W(a, x), W(a,-x)$ and their first derivatives; it is important to 
Figure 3: Regions in the $(x, a)$-plane $(a>0)$ where the different approximations can be used in the computation of the $W(a, x)$ function for an accuracy better than $5 \times 10^{-13}$. 
Figure 4: Regions in the $(x, a)$-plane $(a<0)$ where the different approximations can be used in the computation of the $W(a, x)$ function for an accuracy better than $5 \times 10^{-13}$. 
observe that local Taylor series compute $W(a, x)$ (and $W(a,-x)$ ) and the first derivative not only for one value of $x$ but for several values (in our implementation, 640 values of both $W(a, x)$ and its derivative at regularly spaced $x$-points in the interval $[10,50]$ are obtained). For $a=2$ and $x=2$, where Maclaurin series are used, Maple takes $0.056 s$ for computing $W(a, x)$ and the Fortran program takes $0.059 s$ for computing $W(a, x), W(a,-x)$ and the derivatives. It is very likely that both programs do the same computation (Maclaurin series) in this last case, which explains why the times spent are not very different. It appears that Maple keeps this series as the sole method of computation, which explains why it becomes so slow for larger values of the parameters.

\section{Acknowledgements}

The authors thank the referee for careful reading the manuscript and valuable comments. The authors acknowledge financial support from Ministerio de Educación y Ciencia, project MTM2006-09050 and Ministerio de Ciencia e Innovación, project MTM2009-11686. NMT acknowledges financial support from Gobierno of Navarra, Res. 07/05/2008. 